\title{
Complex Formation of Poly(4-vinylpyridine) with Copper(II) Ion in Mixed Solvent
}

\author{
Akira NAgAi, Akio TAKahashi, Tsuyoshi Komatsu,* \\ and Tsurutaro NAKAGAWA* \\ Hitachi Research Laboratory, Hitachi Ltd., \\ Kuji-cho, Hitachi, Ibaraki 319-12, Japan \\ * Department of Polymer Science, Faculty of Science, \\ Hokkaido University, Kita-ku, Sapporo, \\ Hokkaido 060, Japan
}

(Received March 2, 1988)

\begin{abstract}
Complex formation of poly(4-vinylpyridine) with $\mathrm{Cu}(\mathrm{II})$ ion in a mixed solvent (water-methanol) was investigated potentiometrically, viscometrically, and spectroscopically. Poly(4-vinylpyridinium chloride) was observed to undergo a $\mathrm{pH}$-induced conformational transition. Adding neutral salt caused a reduction in electrostatic repulsion among charges on the polymer chain. The stability of compact form was low, due to hydrophobic interactions among stacked pyridine rings. The maximum average coordination number was nearly three, in spite of the bulky ligand. The polymer chain was constricted by intramolecular complex formation since no charge exists on the ligands. The polymer-metal complex formation was not a stepwise reaction, but a one-step cooperative reaction resulting from the chelate effect of polymer ligands. Formation of an intramoleculer complex for each $\mathrm{Cu}(\mathrm{II})$ ion required nine or ten ligand units on the polymer chain, owing to the rigidity of polymer chain and the steric hindrance of bulky pyridine rings.
\end{abstract}

KEY WORDS Polymer-Metal complex / Polyelectrolyte / Mixed Solvent / Potentiometric Titration / Viscosity /

Extensive literature is available on the formation and coordination number of polymermetal complexes in aqueous solution. Fourcoordinate square-planar structures are known for $\mathrm{Cu}(\mathrm{II})$ ion with poly(vinylamine). ${ }^{1,2}$ Formation of the four-coordinate complex is easily accomplished since their ligands are small and no steric hindrance is encountered. On the other hand, $\mathrm{Cu}(\mathrm{II})$ ion forms twocoordinate complexes with poly(acrylic acid), which has a negative charge and bulky structure. ${ }^{3}$ Its complex formation is affected by electrostatic repulsion and steric hindrance among ligands. Although poly(4-vinylpyridine) (PVP) is a bulky ligand, it is reported to give four-coordinate complexes with $\mathrm{Cu}(\mathrm{II})$ ion in aqueous solution. ${ }^{4}$ Any quantitative esti- mation of the complex formation is however very difficult since PVP does not dissolve into water. The application of the mixed solvent has therefore been attempted for the analyses of polymer-metal complex formation. ${ }^{5}$

In this paper, we studied the complex formation of PVP with $\mathrm{Cu}(\mathrm{II})$ ion in the watermethanol mixed solvent. The solvent provides sufficient solubility of PVP to allow potentiometric, viscometric, and spectroscopic measurements.

\section{EXPERIMENTAL}

\section{Materials}

All the chemicals used were of reagent grade. Poly(4-vinylpyridine) (PVP) was pre- 


\section{A. NAGAl et al.}

pared by the radical polymerization of 4vinylpyridine monomer. The monomer was first purified by distillation in vacuo. The polymerization was carried out at $70^{\circ} \mathrm{C}$ for $10 \mathrm{~h}$ in methanol (volume ratio monomersolvent $=1: 2$ ) using 2,2'-azobisisobutyronitrile as initiator. The product was purified by reprecipitation with diethyl ether-methanol mixture and freeze-dried with $t$-butanol. The degree of polymerization was about 1000 based on a viscometric measurement in ethanol. ${ }^{6}$

All the reagents used as stock solutions were dissolved in the mixture of water and methanol (volume ratio $1: 1$ ). Carbonate-free sodium hydroxide stock solution (concentration: $0.1 \mathrm{moll}^{-1}$ ) was used as a titrant. The concentration of cupric nitrate stock solution was determined by chelatometric titration with 1pyridylazo-2-naphthol (PAN) as indicator.

\section{Potentiometric Titration}

The $\mathrm{pH}$ measurements were carried out using a digital $\mathrm{pH}$ meter (Toa Electronics, Model HM12A). The titrations were carried out in a titration vessel under a nitrogen atmosphere in order to eliminate effect of carbonate. The vessel was placed in a water jacket maintained at $25.0 \pm 0.1{ }^{\circ} \mathrm{C}$. A salt bridge of saturated potassium nitrate agar was used between a glass electrode and a reference calomel electrode. The solvent for all titrations was water-methanol (volume ratio $1: 1$ ) mixture. The concentration of hydrogen ion in the solution was calculated from a calibration curve obtained by the titration of hydrogen chloride in the same mixed solvent. ${ }^{7}$

\section{Viscometric Measurement}

The reduced viscosities of complex solutions were measured with an Ubbelohde viscometer at $25.0 \pm 0.1{ }^{\circ} \mathrm{C}$, in the course of titration with a sodium hydroxide solution under a nitrogen atmosphere.

\section{Spectroscopic Measurement}

The visible spectra of complex solutions were measured by a digital double beam spec-

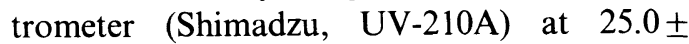
$0.1^{\circ} \mathrm{C}$. The solvent was pure dimethylsulfoxide (DMSO). DMSO is a suitable solvent for PVP and PVP-Cu complexes. Poly (4vinylpyridinium chloride) is insoluble in DMSO, and thus DMSO cannot be used for potentiometric and viscometric titrations.

\section{RESULTS AND DISCUSSION}

The Dissociation Constant of Poly(4-vinylpyridinium chloride) ( $\mathrm{PVPHCl})$

The apparent dissociation constant $\left(\mathrm{p} K_{\mathrm{a}}\right)$ of $\mathrm{PVPHCl}$ in a mixed solvent is shown in Figure 1. The mixed solvent consists of water and methanol (volume ratio $1: 1$ ). The $\mathrm{p} K_{\mathrm{a}}$ increased with the degree of dissociation $(\alpha)$. This was attributed to the charge density on polymer chain which changed with $\alpha$. The charge density on polymer chain was very high at low $\alpha$, so the hydrogen ion was easily dissociated owing to the electrostatic repulsion among charges. The acidity of polyelectrolyte was stronger than that of pyridinium ion at low $\alpha$. This repulsion was lowered at high $\alpha$. The acidity of polymer finally approached that of pyridinium ion $\left(\mathrm{p} K_{\mathrm{a}}=5\right)$. Adding potassium nitrate as a neurtal salt, the acidity of polymer

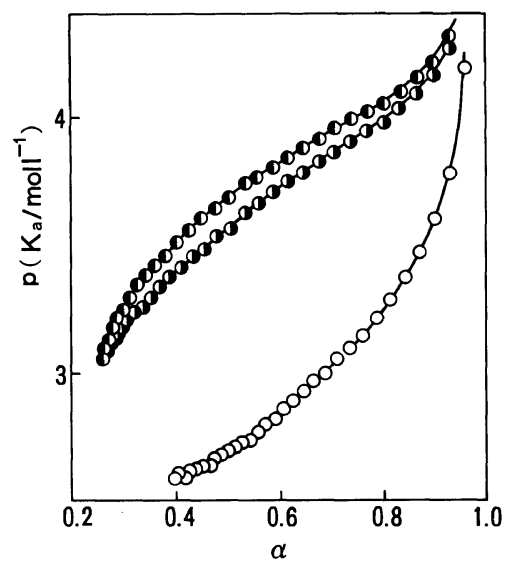

Figure 1. Apparent dissociation constant of $\mathrm{PVPHCl}$ $\left(1.00 \times 10^{-2} \mathrm{moll}^{-1}\right)$ at different ionic strengths $\left(\mathrm{KNO}_{3}\right): \bigcirc, 0 ; 0,0.1 ; \mathbf{D}, 0.2$. 
became weak, since charge on the polymer chain would be shielded by the neutral salt and the electrostatic repulsion would be reduced. The $\mathrm{p} K_{\mathrm{a}}$ increased continuosly with $\alpha$, in the absence of any neutral salt, but a slight conformational transition of the polymer chain was observed when any neutral salt was present. In the case of PVPHCl, the chain was in a state of extended form at low $\alpha$ and in a compact from at high $\alpha(\alpha>0.7)$. As shown in Figure 1, the stability of the compact form was very low. The compact form has been attributed to the hydrophobic interaction of methyl groups in poly(methacrylic acid). ${ }^{8}$ The driving force for the compact form in the present case seemed to be the stacking interaction of pyridine rings in the PVPHCl. The decrease in Gibbs free energy by this stacking interaction was far less than the increase of that by the electrostatic repulsion in the absence of neutral salt. By the addition of neutral salt, this electrostatic repulsion became weak and was compensated by the stacking interaction. Therefore the conformational transition appeared to arise from a balance between the two above-mentioned interactions.

Based on the $\alpha$ value, $40 \%$ of the hydrogen ions were already dissociated before the neutralization in the absence of any neutral salt. There was about a $25 \%$ hydrogen ion dissociation when neutral salt was present. This was attributed to the effect of charge shielding on the chain by the neutral salt. No precipitation occurred during the acid-base titration of $\mathrm{PVPHCl}$ using the water-methanol mixed solvent.

\section{Potentiometric Titration of Complex Solution}

The $\mathrm{pH}$ titration curves of $\mathrm{PVPHCl}$ in the presence and the absence of $\mathrm{Cu}$ (II) ion are shown in Figure 2. The numbers on ordinate represent the reduced values of concentration of hydrogen ion, $-\log \left([\mathrm{H}] / \mathrm{moll}^{-1}\right)$. These values were obtained from the calibration curve of relationship between the electromotive force and the concentration of hydrogen ion, assum- ing complete dissociation of hydrogen chloride in the mixed solvent. This method was available when neutral salt was present, provided that the ionic strength was constant throughout the titration. But it was not suitable when the ionic strength changed (e.g., in the absence of neutral salt). In the presence of $\mathrm{Cu}$ (II) ion, the complex formation occurred as an ex-

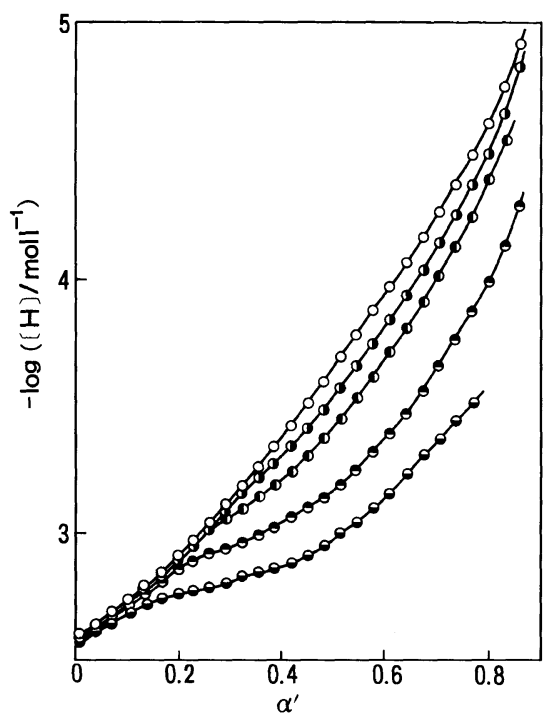

Figure 2. Titration curves of $\mathrm{PVPHCl}\left(1.00 \times 10^{-2}\right.$ moll $\left.{ }^{-1}\right)$ with $\mathrm{Cu}(\mathrm{II})$ ion $\left(\times 10^{-3} \mathrm{moll}^{-1}\right): 0,0 ; 0$, $0.269(R=40)$; D, $0.538(20) ; \ominus, 1.08(10) ; \ominus, 2.15(5)$ all at ionic strength of $0.1\left(\mathrm{KNO}_{3}\right)$.

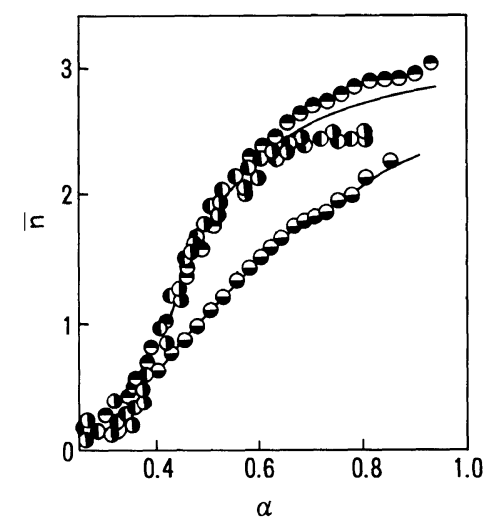

Figure 3. Formation curves of PVPHCl $\left(1.00 \times 10^{-2}\right.$ mol $\left.1^{-1}\right)$ with $\mathrm{Cu}(\mathrm{II})$ ion $\left(\times 10^{-3} \mathrm{moll}^{-1}\right)$ :, 0.269 $(R=40) ; 0,0.538(20) ; \Theta, 1.08(10) ; \ominus, 2.15(5)$ all at ionic strength of $0.1\left(\mathrm{KNO}_{3}\right)$. 
change reaction of hydrogen ion with $\mathrm{Cu}(\mathrm{II})$ ion, accompanied by a decrease in the value of $-\log \left([\mathrm{H}] / \mathrm{moll}^{-1}\right)$. The value of degree of neutralization $\left(\alpha^{\prime}\right)$ where the titration curve disconnected from that of the $\mathrm{Cu}$ (II)-free solution increased with the increase in the concentration ratio of ligand to metal ion $(R)$ as shown in Figure 2.

The average coordination number $(\bar{n})$ obtained from the $\mathrm{pH}$ titration curve is shown in Figure 3. Calculation of $\bar{n}$ was done using the method of reference plot contrived by Mandel et al. ${ }^{9}$ The formation curves were almost identical being independent of $R$ above 10. The value of $\bar{n}$ was close to three at high $\alpha$. Although PVP is a bulky ligand, the complex formed high-coordinate species. This was due to the fact that PVP had no charge and electrostatic repulsion did not occur on complex formation, unlike the case of poly(acrylic acid), which is a ligand with negative charges.

As shown in Figure 3, the value of $\alpha$ at the commencement of complex formation was independent of $R$, whereas the value of $\alpha^{\prime}$ at the commencement of formation function curve was dependent on $\mathrm{R}$ as shown in Figure 2.

When $R$ was 5 , the formation curve differed from those of the other $R$ values. This suggests that five ligand units are insufficient for the formation of intramolecular complex. So in this case, precipitation occurred at high $\alpha$ since the formation of intermolecular complex probably took place. This agrees with the results of molar ratio measurements to be mentioned later.

\section{Viscosity of Complex Solutions}

The reduced viscosities of $\mathrm{PVPHCl}$ solutions in the presence and absence of metal ion are shown in Figure 4. The viscosity of $\mathrm{Cu}$ (II)-free PVPHCl solution had a constant value until $\alpha^{\prime}$ reached 0.25 , and then lowered as $\alpha^{\prime}$ became high. The reason for this was that $25 \%$ of the hydrogen ions was already dissociated before neutralization as shown in Figure 1 . The hydroxide ion did not react with

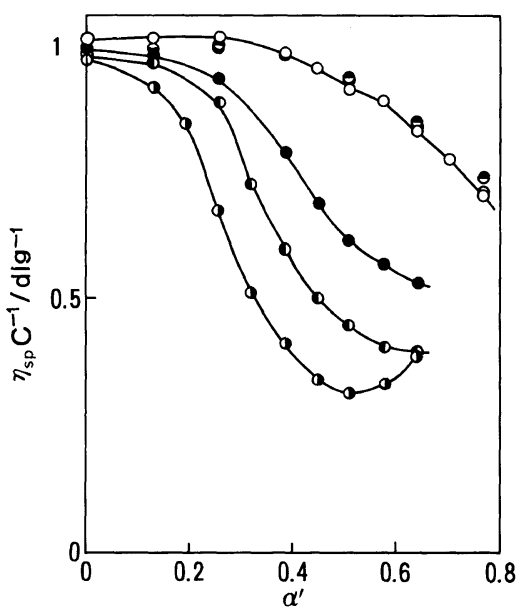

Figure 4. Reduced viscosities of PVPHCl $(1.00 \times$ $10^{-2} \mathrm{~mol}^{-1}$ ) solutions in the absence of metal ion (O) and in the presence of $\mathrm{Cu}(\mathrm{II})$ ion $\left(\times 10^{-3}\right.$ mol $\left.l^{-1}\right) ; 0,0.269(R=40) ; \bigcirc, 0.538(20) ; 0,1.08$ (10) $\mathrm{Ca}$ (II) ion $\left(\times 10^{-3} \mathrm{~mol} \mathrm{l}^{-1}\right)$; $\Theta, 0.512$ (40); $\Theta$, $1.02(10)$ all at ionic strength of $0.1\left(\mathrm{KNO}_{3}\right)$.

the hydrogen ion bonded to the polymer chain, but rather with that of dissociated ion until $\alpha^{\prime}$ reached 0.25 . Therefore, the conformation of chain did not change, since charge density on the polymer chain was constant. When $\alpha^{\prime}$ was above 0.25 , the viscosity went down owing to the decrease in electrostatic repulsion among the pyridinium groups and polymer chain shrinkage.

This constriction of the polymer chain was distinctive in the presence of $\mathrm{Cu}(\mathrm{II})$ ion. It was due to formation of an intramolecular complex in which several ligands of the same chain were coordinated to the $\mathrm{Cu}$ (II) ion. The polymer chain was not changed by addition of $\mathrm{Ca}$ (II) ion which does not form complexes with PVP.

The decrease in viscosity depended on $R$. The intramolecular complex could therefore consume many ligands per a metal ion at high $R$.

On decreasing viscosity, the value of $\alpha^{\prime}$ was dependent on $R$. The lower $R$ was, the lower $\alpha^{\prime}$ was. This agrees with the relationship between $R$ and $\alpha^{\prime}$ of titration curves from that of 
$\mathrm{Cu}$ (II)-free $\mathrm{PVPHCl}$ solution as shown in Figure 2.

The reason for the increase in viscosity at high $\alpha^{\prime}$ when $R$ was 10 is not obvious. The formation of intermolecular complex may possibly be involved.

\section{Visible Spectra of Complex Solutions}

Spectrophotometric measurements were carried out with varying the concentration of ligand at a constant metal ion concentration, $1.04 \times 10^{-2} \mathrm{~mol} \mathrm{l}^{-1}$. The results are shown in Figure 5. The solvent used here was pure dimethylsulfoxide (DMSO) which is suitable for PVP and PVP-Cu(II) complexes. The maximum absorption of $\mathrm{Cu}(\mathrm{II})$ ion in DMSO was observed at $830 \mathrm{~nm}$, similar to that of $\mathrm{Cu}(\mathrm{II})$ ion in water (aqua complex) as shown in

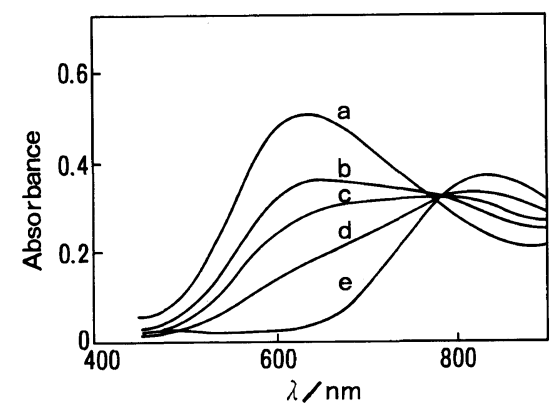

Figure 5. Visible spectra of $\mathrm{Cu}(\mathrm{II})$ ion $\left(1.04 \times 10^{-2}\right.$ mol $\left.1^{-1}\right)$ with PVP in DMSO. The concentration ratio $(R=[\mathrm{PVP}] /[\mathrm{Cu}])$ are $9.81(\mathrm{a}), 5.60(\mathrm{~b}), 4.04$ (c), $2.26(\mathrm{~d}), 0$ (e).

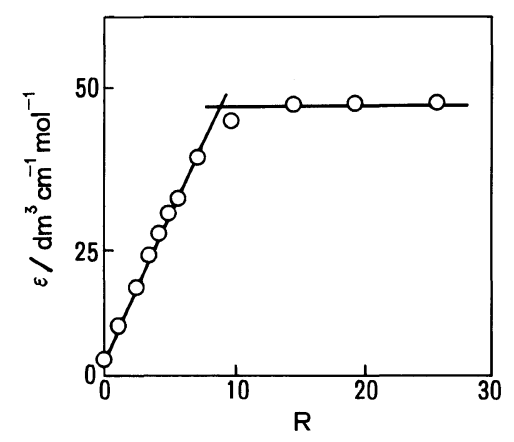

Figure 6. Molar extinction coefficient of $\mathrm{Cu}(\mathrm{II})$ complex solutions $\left(1.04 \times 10^{-2} \mathrm{moll}^{-1}\right)$ at $630 \mathrm{~nm}$ as a function of $R$.
Figure 5(e). As R became high, the absorbance at $830 \mathrm{~nm}$ was reduced and that at $630 \mathrm{~nm}$ increased. There were only two complex species, considering that the wave length of absorption peak was independent of $R$ and one isosbestic point was observed at $780 \mathrm{~nm}$. The transition of $\left[\mathrm{Cu}(\mathrm{DMSO})_{x}\right]$ to $\left[\mathrm{Cu}(\mathrm{VP})_{y}\right]$ was presumed to occur ( $\mathrm{VP}=$ pyridine ligand unit). This agrees with published reports that at the same $\mathrm{pH}$, the only one kind of PVP complex species forms with $\mathrm{Cu}(\mathrm{II})$ ion which is independent of $R .^{10-12}$ Formation of such a highly coordinated complex species was due to the socalled polymer effect. As the complex formation reaction is generally a stepwise reaction in the case of low molecular ligand, the average coordination number depends on $R$. On the other hand, in the case of polymeric ligand, one ligand coordinates with the metal ion and adjacent ligands on the same polymer chain are then fixed near this metal ion. The local concentration of ligand near this metal ion is higher than that in the absence of metal ion. A highly coordinated complex is much stabler than that of a low-coordinated one.

The change in molar extinction coefficient $(\varepsilon)$, at $630 \mathrm{~nm}$, with $R$ is shown in Figure 6. The $\varepsilon$ reached a constant value, when $R$ reached 9 or 10 . In the case of polymeric ligand, the formation of intramolecular complex needs futile ligands most of which are not able to coordinate since the ligands are fixed on a rigid polymer chain. The results obtained by the molar ratio method do not indicate the average coordination number $(\bar{n})$, but rather the number of ligands required for the formation of intramolecular complex. It was assumed that the high coordinate structure was made up of 9 or 10 ligand units and one metal ion. This agrees with the result of viscosity measurement reported by Tsuchida et al. ${ }^{13}$

\section{Solubility of Polymer-Metal Complexes}

Solubility of the polymer-metal complex was dependent on the $R$ and the ionic strength of solutions. The solubility decreased as the con- 


\section{A. NAGAI et al.}

centration of neutral salt increased. Polymer chains were then close to each other, since the electrostatic repulsion among chains was reduced by shielding of charges on chains owing to the neutral salt. On the other hand, at high $\alpha$, the charge density on chain was low. The intermolecular complex in which the polymer chains cross-linked by $\mathrm{Cu}$ (II) ion was formed through rearrangement of ligands in the intramolecular complex. As the concentration of metal ion became high, the probability of many polymer chains existing near a metal ion increased and the intermolecular complex was easily formed. Consequently the solubility of complexes became low.

\section{CONCLUSIONS}

Good $\mathrm{pH}$ titration curves were obtained for poly(vinylpyridinium chloride) (PVPHCl) in the mixed solvent. When the electrostatic repulsion was reduced by adding any neutral salt, the conformational transition was observed since the repulsion was compensated by the hydrophobic interaction of pyridine rings.

When $R$ was over 10 , the complex formation curve had the same dependence on the charge density of chain. The average coordination number was about three. Poly(vinylpyridine) formed a high-coordination number complex with $\mathrm{Cu}(\mathrm{II})$ in spite of the bulky ligand. The cause of this was the lack of charge on the ligand by the coordination. The constriction of polymer chain was enhanced by the intramo- lecular complex formation. The complex formation reaction of PVP was not stepwise, as in the case of the monomeric ligand, but occurred in one-step in which a non-coordinated species became highly coordinated all at once. This was due to the chelate effect of polymer ligands. According to the results by molar ratio method, it was found that the intramolecular complex needed at least nine or ten ligand units per a metal ion owing to the rigidity of polymer chain and the steric hindrance of bulky pyridine rings.

\section{REFERENCES}

1. Ph. Teyssie, C. Decoene, and M. T. Teyssie, Makromol. Chem., 84, 51 (1965).

2. K. Kimura, Y. Inaki, and K. Takemoto, Makromol. Chem., 175, 83 (1974).

3. F. Yamashita, T. Komatsu, and T. Nakagawa, Bull. Chem. Soc. Jpn., 49, 2073 (1976).

4. H. Nishikawa and E. Tsuchida, J. Phys. Chem., 79, 2072 (1975).

5. A. Nagai, T. Komatsu, and T. Nakagawa, Polym. Prepr. Jpn., 32, 500 (1983).

6. J. B. Berkowitz, M. Yamin, and R. M. Fuoss, J. Polym. Sci., 28, 69 (1958).

7. H. Ohtaki, Kagaku no Ryoiki, 21, 3 (1967).

8. M. Prandny, I. Kminek, and S. Sevcik, Polym. Bull., 16, 195 (1986).

9. M. Mandel and J. C. Leyte, J. Polym. Sci., A, 2, 2883 (1964).

10. H. Nishide and E. Tsuchida, J. Polym. Sci., Chem. Ed., 19, 835 (1981).

11. Yu. I. Skurlatov, A. I. Kokorin, S. O. Travin, Yu. E. Kirsh and A. P. Purmal, Polym. Sci., USSR, 24, 2143 (1982).

12. A. S. Polinskii, V..S. Pshezhetskii, and V. A. Kabanov, Polym. Sci., USSR, 27, 2577 (1985).

13. H. Nishide and E. Tsuchida, Makromol. Chem., 177, 2295 (1976). 